\title{
In situ powder X-ray crystallography for gas sorption in metal- organic frameworks
}

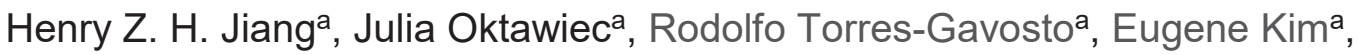 \\ Benjamin A. Trump ${ }^{b}$, Craig M. Brown ${ }^{b, c}$, Jeffrey R. Long ${ }^{a, d, e}$ \\ aDepartment of Chemistry, University of California, Berkeley, California, 94720, USA; \\ h.jiang@berkeley.edu \\ ${ }^{b}$ National Institute of Standards and Technology, Center for Neutron Research, \\ Gaithersburg, Maryland 20899, USA
}

${ }^{\circ}$ Chemical and Biomolecular Engineering, University of Delaware, Newark, Delaware 19716, USA

dDepartment of Chemical and Biomolecular Engineering, University of California, Berkeley, California, 94720, USA

eMaterials Science Division, Lawrence Berkeley National Laboratory, Berkeley, California, 94720, USA

Metal-organic frameworks are a class of porous materials with attractive features for applications such as gas storage, gas separations, sensing and catalysis. As these frameworks are crystalline, X-ray crystallography is a key analytical technique and is often employed to examine structure-property relationships in such materials. In situ X-ray crystallography expands upon this powerful technique by allowing these materials to be probed under a variety of temperatures, pressures and atmospheres, yielding structural information crucial in elucidating sorbate-sorbent interactions and the mechanism of gas binding. Recently, we have synthesized several frameworks which bind gases through reactivity at metal centers by mechanisms such as electron transfer and cooperative chemisorptive insertion. We have studied these materials using in situ powder x-ray diffraction, demonstrating the versatility and efficacy of this technique in the characterization of porous materials. 